Pacific

Journal of

Mathematics

COCHARACTER-CLOSURE AND SPHERICAL BUILDINGS

Michael Bate, Sebastian Herpel, BENJAMIN MARTIN AND GERHARD RÖHRLE

Volume $279 \quad$ No. 1-2

December 2015 


\title{
COCHARACTER-CLOSURE AND SPHERICAL BUILDINGS
}

\author{
Michael Bate, Sebastian Herpel, \\ BENJAMIN MARTIN AND GERHARD RÖHRLE
}

In memory of Robert Steinberg

\begin{abstract}
Let $\boldsymbol{k}$ be a field, let $\boldsymbol{G}$ be a reductive $\boldsymbol{k}$-group and $\boldsymbol{V}$ an affine $\boldsymbol{k}$-variety on which $G$ acts. In this note we continue our study of the notion of cocharacterclosed $G(k)$-orbits in $V$. In earlier work we used a rationality condition on the point stabilizer of a $G$-orbit to prove Galois ascent/descent and Levi ascent/descent results concerning cocharacter-closure for the corresponding $G(k)$-orbit in $V$. In the present paper we employ building-theoretic techniques to derive analogous results.
\end{abstract}

\section{Introduction}

Let $k$ be a field and let $G$ be a reductive linear algebraic group acting on an affine variety $V$, with $G, V$ and the action all defined over $k$. Let $\Delta_{k}$ be the (simplicial) spherical building of $G$ over $k$, and let $\Delta_{k}(\mathbb{R})$ be its geometric realisation (for precise definitions, see below). In this paper we continue the study, initiated in [Bate et al. 2013; 2012; 2015], of the notion of cocharacter-closed orbits in $V$ for the group $G(k)$ of $k$-rational points of $G$, and of interactions with the geometry of $\Delta_{k}(\mathbb{R})$. The philosophy of this paper is as follows (cf. [Bate et al. 2015]): for a point $v$ in $V$, we are interested in Galois ascent/descent questions - given a separable algebraic extension $k^{\prime} / k$ of fields, how is the $G\left(k^{\prime}\right)$-orbit of $v$ related to the $G(k)$-orbit of $v$ ? - and Levi ascent/descent questions - given a $k$-defined torus $S$ of the stabilizer $G_{v}$, how is the $C_{G}(S)(k)$-orbit of $v$ related to the $G(k)$-orbit of $v$ ? (See [Bate et al. 2015, Section 5, Paragraph 1] for an explanation of the terms Galois/Levi ascent/descent in this context.) These questions are related, and have natural interpretations in $\Delta(\mathbb{K})$.

Our results complement those of [Bate et al. 2015]: they give similar conclusions but under different assumptions. It was shown in [loc. cit.] (see Proposition 2.6 below) that Galois descent — passing from $G\left(k^{\prime}\right)$-orbits to $G(k)$-orbits — is always

MSC2010: primary 20G15; secondary 14L24.

Keywords: Affine $G$-variety, cocharacter-closed orbit, rationality, spherical building, the centre conjecture. 
well-behaved. Certain results on Galois ascent were also proved [loc. cit., Theorem 5.7] under hypotheses on the stabilizer $G_{v}$. The mantra in this paper is that when the centre conjecture (see Theorem 1.2 below) is known to hold, one can use it to prove Galois ascent results, and hence deduce Levi ascent/descent results. The idea is that when the extension $k^{\prime} / k$ is separable and normal, questions of Galois ascent can be interpreted in terms of the action of the Galois group of $k^{\prime} / k$ on the building; moreover, if one has such Galois ascent questions under control, then it is easier to handle Levi ascent/descent because one may assume that the torus $S$ is split (cf. [loc. cit., Theorem 5.4(ii)]).

When $k$ is algebraically closed (or more generally when $k$ is perfect), our setup is also intimately related to the optimality formalism of [Kempf 1978; Rousseau 1978; Hesselink 1978]. Indeed, one may interpret this formalism in the language of the centre conjecture (see [Bate et al. 2012, Section 1]). The idea is to study the $G$-orbits in $V$ via limits along cocharacters of $G$ : limits are formally defined below, but given $v$ in $V$, if we take the set of cocharacters $\lambda$ of $G$ for which the

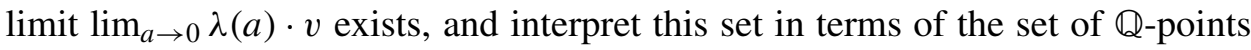
$\Delta(\mathbb{Q})$ of the building of $G$, then we obtain a convex subset $\Sigma_{v}$ of $\Delta(\mathbb{Q})$. In case $G \cdot v$ is not Zariski-closed, one can find a fixed point in the set $\Sigma_{v}$ and an associated optimal parabolic subgroup $P$ of $G$ with many nice properties: in particular, the stabilizer $G_{v}$ normalises $P$. It is not currently known in general how to produce analogues of these optimality results over arbitrary fields (or even whether such results exist); see [Bate et al. 2013, Section 1] for further discussion. Our first main theorem gives a rational analogue of the Kempf-Rousseau-Hesselink ideas when $\Sigma_{v, k_{s}}$ (the points of $\Sigma_{v}$ coming from $k_{s}$-defined cocharacters of $G$ ) happens to be a subcomplex of $\Delta(\mathbb{Q})$, and also answers in this case the ascent/descent questions posed earlier.

Theorem 1.1. Let $v \in V$. Suppose $\Sigma_{v, k_{s}}$ is a subcomplex of $\Delta_{k_{s}}(\mathbb{Q})$. Then the following hold:

(i) Suppose $v \in V(k)$ and $G\left(k_{s}\right) \cdot v$ is not cocharacter-closed over $k_{s}$. Let $S$ be any $k$-defined torus of $G_{v}$ and set $L=C_{G}(S)$. Then there exists $\sigma \in Y_{k}(L)$ such that $\lim _{a \rightarrow 0} \sigma(a) \cdot v$ exists and lies outside $G\left(k_{s}\right) \cdot v$.

(ii) Suppose $v \in V(k)$. For any separable algebraic extension $k^{\prime} / k, G\left(k^{\prime}\right) \cdot v$ is cocharacter-closed over $k^{\prime}$ if and only if $G(k) \cdot v$ is cocharacter-closed over $k$.

(iii) Let $S$ be any $k$-defined torus of $G_{v}$ and set $L=C_{G}(S)$. Then $G(k) \cdot v$ is cocharacter-closed over $k$ if and only if $L(k) \cdot v$ is cocharacter-closed over $k$.

The hypothesis that $\Sigma_{v, k_{s}}$ is a subcomplex allows us to apply the following result — Tits' centre conjecture — in the proof of Theorem 1.1: 
Theorem 1.2. Let $\Theta$ be a thick spherical building and let $\Sigma$ be a convex subcomplex of $\Theta$ such that $\Sigma$ is not completely reducible. Then there is a simplex of $\Sigma$ that is fixed by every building automorphism of $\Theta$ that stabilizes $\Sigma$. (We call such a simplex a centre of $\Sigma$.)

For definitions and further details, see [Ramos-Cuevas 2013]; in particular, note that the spherical building of a reductive algebraic group is thick. The conjecture was proved by Mühlherr and Tits [2006], Leeb and Ramos-Cuevas [2011] and Ramos-Cuevas [2013], and a uniform proof for chamber subcomplexes has also now been given by Mühlherr and Weiss [2013]. The condition that $\Sigma_{v, k_{s}}$ is a subcomplex is satisfied in the theory of complete reducibility for subgroups of $G$ and Lie subalgebras of $\operatorname{Lie}(G)$, and our results yield applications to complete reducibility (see Theorem 1.4 below).

By [Bate et al. 2015, Theorem 5.7], the conclusions of Theorem 1.1(ii) and (iii) hold if $G_{v}$ has a maximal torus that is $k$-defined. Our second main result gives alternative hypotheses on $G_{v}$, this time of a group-theoretic nature, for the conclusions of Theorem 1.1 to hold, without the assumption that $\Sigma_{v, k_{s}}$ is a subcomplex. The proof of this result relies in an essential way on known cases of a strengthened version of the centre conjecture (this time from [Bate et al. 2012]).

Theorem 1.3. Let $v \in V(k)$. Suppose that $(a) G_{v}^{0}$ is nilpotent, or $(b)$ every simple component of $G^{0}$ has rank 1 . Then the following hold:

(i) Suppose $G\left(k_{s}\right) \cdot v$ is not cocharacter-closed over $k_{s}$. Let $S$ be any $k$-defined torus of $G_{v}$ and set $L=C_{G}(S)$. Then there exists $\sigma \in Y_{k}(L)$ such that $G_{v}\left(k_{s}\right)$ normalises $P_{\sigma}\left(G^{0}\right)$ and $\lim _{a \rightarrow 0} \sigma(a) \cdot v$ exists and lies outside $G\left(k_{s}\right) \cdot v$.

(ii) For any separable algebraic extension $k^{\prime} / k, G\left(k^{\prime}\right) \cdot v$ is cocharacter-closed over $k^{\prime}$ if and only if $G(k) \cdot v$ is cocharacter-closed over $k$.

(iii) Let $S$ be any $k$-defined torus of $G_{v}$ and set $L=C_{G}(S)$. Then $G(k) \cdot v$ is cocharacter-closed over $k$ if and only if $L(k) \cdot v$ is cocharacter-closed over $k$.

The hypothesis in Theorem 1.1(i) that $v$ is a $k$-point ensures that the subset $\Sigma_{v}$ is Galois-stable, and it is also needed in our proof of Theorem 1.3 (but see Remark 4.6). Sometimes, however, one can get away with a weaker hypothesis. This happens for $G$-complete reducibility in the final section of the paper, where we prove the following ascent/descent result:

Theorem 1.4. Suppose that $G$ is connected. Let $H$ be a subgroup of $G$. Let $S$ be a $k$-defined torus of $C_{G}(H)$ and set $L=C_{G}(S)$. Then $H$ is $G$-completely reducible over $k$ if and only if $H$ is L-completely reducible over $k$.

Remark 1.5. (i) Theorem 1.4 gives an alternative proof and also slightly generalises Serre's Levi ascent/descent result [Serre 1997, Proposition 3.2]; cf. [Bate et al. 2005, 
Corollary 3.21, Corollary 3.22] — for in the statement of Theorem 1.4, we do not require $H$ to be a subgroup of $G(k)$.

(ii) The counterpart of Theorem 1.1(ii) (Galois ascent/descent for $G$-complete reducibility) was proved in [Bate et al. 2009].

We spend much of the paper recalling relevant results from geometric invariant theory and the theory of buildings. Although the basic ideas are familiar, we need to extend many of them: for instance, the material on quasi-states in Section 3D was covered in [Bate et al. 2012] for algebraically closed fields, but we need it for arbitrary fields. We work with the geometric realisations of buildings rather than with buildings as abstract simplicial complexes; some care is needed when the reductive group $G$ has positive-dimensional centre.

The paper is laid out as follows. In Section 2, we set up notation and collect terminology and results relating to cocharacter-closedness. In Section 3, we translate our setup into the language of spherical buildings; we use notation and results from [Bate et al. 2012] on buildings, some of which we extend slightly. In Section 4, we combine the technology from both of the preceding sections to give proofs of our main results. In the final section we give our applications to the theory of complete reducibility.

\section{Notation and preliminaries}

Let $k$ denote a field with separable closure $k_{s}$ and algebraic closure $\bar{k}$. Let $\Gamma:=$ $\operatorname{Gal}\left(k_{s} / k\right)=\operatorname{Gal}(\bar{k} / k)$ denote the Galois group of $k_{s} / k$. Throughout, $G$ denotes a (possibly nonconnected) reductive linear algebraic group defined over $k$, and $V$ denotes a $k$-defined affine variety upon which $G$ acts $k$-morphically. Let $G(k)$, $G\left(k_{s}\right), V(k), V\left(k_{s}\right)$ denote the $k$ - and $k_{s}$-points of $G$ and $V$; we usually identify $G$ with $G(\bar{k})$ and $V$ with $V(\bar{k})$. If $X$ is a variety then we denote its Zariski closure by $\bar{X}$.

More generally, we need to consider $k$-points and $k_{s}$-points in subgroups that are not necessarily $k$-defined or $k_{s}$-defined; note that if $k$ is not perfect then even when $v$ is a $k$-point, the stabilizer $G_{v}$ need not be $k$-defined. If $k^{\prime} / k$ is an algebraic field extension and $H$ is a closed subgroup of $G$ then we set $H\left(k^{\prime}\right)=H(\bar{k}) \cap G\left(k^{\prime}\right)$, and we say that a torus $S$ of $H$ is $k^{\prime}$-defined if it is $k^{\prime}$-defined as a torus of the $k$-defined group $G$. Note that a $k_{s}$-defined torus of $H$ is a torus of $\overline{H\left(k_{s}\right)}$.

2A. Cocharacters and $\boldsymbol{G}$-actions. Given a $k$-defined algebraic group $H$, we let $Y(H)$ denote the set of cocharacters of $H$, with $Y_{k}(H)$ and $Y_{k_{s}}(H)$ denoting the sets of $k$-defined and $k_{s}$-defined cocharacters, respectively. The group $H$ acts on $Y(H)$ via the conjugation action of $H$ on itself. This gives actions of the group of $k$-points $H(k)$ on $Y_{k}(H)$ and the group of $k_{s}$-points $H\left(k_{s}\right)$ on $Y_{k_{s}}(H)$. There is also 
an action of the Galois group $\Gamma$ on $Y(H)$ which stabilizes $Y_{k_{s}}(H)$, and the $\Gamma$-fixed elements of $Y_{k_{s}}(H)$ are precisely the elements of $Y_{k}(H)$. We write $Y=Y(G)$, $Y_{k}=Y_{k}(G)$ and $Y_{k_{s}}=Y_{k_{s}}(G)$.

Definition 2.1. A function \|\|$: Y \rightarrow \mathbb{R}_{\geq 0}$ is called a $\Gamma$-invariant, $G$-invariant norm if:

(i) $\|g \cdot \lambda\|=\|\lambda\|=\|\gamma \cdot \lambda\|$ for all $\lambda \in Y, g \in G$ and $\gamma \in \Gamma$;

(ii) for any maximal torus $T$ of $G$, there is a positive definite integer-valued form ( , ) on $Y(T)$ such that $(\lambda, \lambda)=\|\lambda\|^{2}$ for any $\lambda \in Y(T)$.

Such a norm always exists: To see this, take a $k$-defined maximal torus $T$ and any positive definite integer-valued form on $Y(T)$. Since $T$ splits over a finite extension of $k$, we can average the form over the Weyl group $W$ and over the finite Galois group of the extension to obtain a $W$-invariant $\Gamma$-invariant form on $Y(T)$, which defines a norm satisfying (ii). One can extend this norm to all of $Y$ because any cocharacter is $G$-conjugate to one in $Y(T)$; this procedure is well-defined since the norm on $Y(T)$ is $W$-invariant. See [Kempf 1978] for more details. If $G$ is simple then \|\| is unique up to nonzero scalar multiples. We fix such a norm once and for all.

For each cocharacter $\lambda \in Y$ and each $v \in V$, we define a morphism of varieties $\phi_{v, \lambda}: \bar{k}^{*} \rightarrow V$ via the formula $\phi_{v, \lambda}(a)=\lambda(a) \cdot v$. If this morphism extends to a morphism $\widehat{\phi}_{v, \lambda}: \bar{k} \rightarrow V$, then we say that $\lim _{a \rightarrow 0} \lambda(a) \cdot v$ exists, and set this limit equal to $\widehat{\phi}_{v, \lambda}(0)$; note that such an extension, if it exists, is necessarily unique.

Definition 2.2. For $\lambda \in Y$ and $v \in V$, we say that $\lambda$ destabilizes $v$ provided $\lim _{a \rightarrow 0} \lambda(a) \cdot v$ exists, and if $\lim _{a \rightarrow 0} \lambda(a) \cdot v$ exists and does not belong to $G \cdot v$, then we say $\lambda$ properly destabilizes $v$. We have an analogous notion over $k$ : if $\lambda \in Y_{k}$ then we say that $\lambda$ properly destabilizes $v$ over $k$ if $\lim _{a \rightarrow 0} \lambda(a) \cdot v$ exists and does not belong to $G(k) \cdot v$. Finally, if $k^{\prime} / k$ is an algebraic extension, and $\lambda \in Y_{k}$, then we say that $\lambda$ properly destabilizes $v$ over $k^{\prime}$ if $\lim _{a \rightarrow 0} \lambda(a) \cdot v$ exists and does not belong to $G\left(k^{\prime}\right) \cdot v$; that is, if $\lambda$ - regarded as an element of $Y_{k^{\prime}}(G)$ - properly destabilizes $v$ over $k^{\prime}$.

2B. R-parabolic subgroups. When $V=G$ and $G$ is acting by conjugation, for each $\lambda \in Y$ we get a set $P_{\lambda}:=\left\{g \in G \mid \lim _{a \rightarrow 0} \lambda(a) g \lambda(a)^{-1}\right.$ exists $\}$; this is a parabolic subgroup of $G$. We distinguish these parabolic subgroups by calling them Richardson-parabolic or R-parabolic subgroups. For basic properties of these subgroups, see [Bate et al. 2005, Section 6]. We recall here that $L_{\lambda}=C_{G}(\operatorname{Im}(\lambda))$ is called an $R$-Levi subgroup of $P_{\lambda}, R_{u}\left(P_{\lambda}\right)$ is the set of elements sent to $1 \in G$ in the limit, and $P_{\lambda}=R_{u}\left(P_{\lambda}\right) \rtimes L_{\lambda}$. Further, $R_{u}\left(P_{\lambda}\right)$ acts simply transitively on the set of all $L_{\mu}$ such that $P_{\mu}=P_{\lambda}$ (that is, on the set of all R-Levi subgroups of $\left.P_{\lambda}\right)$ : note that this is a transitive action of $R_{u}\left(P_{\lambda}\right)$ on the set of subgroups of the form $L_{\mu}$, not on the set of cocharacters for which $P_{\mu}=P_{\lambda}$. Most of these things 
work equally well over the field $k$ : for example, if $\lambda$ is $k$-defined then $P_{\lambda}, L_{\lambda}$ and $R_{u}\left(P_{\lambda}\right)$ are; moreover, given any $k$-defined R-parabolic subgroup $P, R_{u}(P)(k)$ acts simply transitively on the set of $k$-defined R-Levi subgroups of $P$ [Bate et al. 2013, Lemma 2.5]. Note that if $P$ is $k$-defined and $G$ is connected then $P=P_{\lambda}$ for some $k$-defined $\lambda$, but this can fail if $G$ is not connected [Bate et al. 2013, Section 2].

When $H$ is a reductive subgroup of $G$ the inclusion $Y(H) \subseteq Y(G)$ means that we get an R-parabolic subgroup of $H$ and of $G$ attached to any $\lambda \in Y(H)$. When we use the notation $P_{\lambda}, L_{\lambda}$, etc., we are always thinking of $\lambda$ as a cocharacter of $G$. If we need to restrict attention to the subgroup $H$ for some reason, we write $P_{\lambda}(H), L_{\lambda}(H)$, etc.

2C. The sets $Y(\mathbb{Q})$ and $Y(\mathbb{R})$. Form the set $Y(\mathbb{Q})$ by taking the quotient of $Y \times \mathbb{N}_{0}$ by the relation $\lambda \sim \mu$ if and only if $n \lambda=m \mu$ for some $m, n \in \mathbb{N}$, and extend the norm function to $Y(\mathbb{Q})$ in the obvious way. For any torus $T$ in $G$, $Y(T, \mathbb{Q}):=Y(T) \otimes_{\mathbb{Z}} \mathbb{Q}$ is a vector space over $\mathbb{Q}$. Now one can form real spaces $Y(T, \mathbb{R}):=Y(T, \mathbb{Q}) \otimes_{\mathbb{Q}} \mathbb{R}$ for each maximal torus $T$ of $G$ and a set $Y(\mathbb{R})$ by glueing the $Y(T, \mathbb{R})$ together according to the way the spaces $Y(T, \mathbb{Q})$ fit together [Bate et al. 2012, Section 2.2]. The norm extends to these sets. One can define sets $Y_{k}(\mathbb{Q}), Y_{k}(\mathbb{R}), Y_{k}(T, \mathbb{Q}), Y_{k}(T, \mathbb{R}), Y_{k_{s}}(\mathbb{Q}), Y_{k_{s}}(\mathbb{R}), Y_{k_{s}}(T, \mathbb{Q})$ and $Y_{k_{s}}(T, \mathbb{R})$ analogously by restricting attention to $k$-defined cocharacters and maximal tori, or $k_{s}$-defined cocharacters and maximal tori, as appropriate. For the rest of the paper, $\mathbb{K}$ denotes either of $\mathbb{Q}$ or $\mathbb{R}$ when the distinction is not important. The sets $Y(\mathbb{K}), Y_{k}(\mathbb{K})$ and $Y_{k_{s}}(\mathbb{K})$ inherit $G$-, $G(k)$-, $G\left(k_{s}\right)$ - and $\Gamma$-actions from those on $Y, Y_{k}$ and $Y_{k_{s}}$, as appropriate, and each element $\lambda \in Y(\mathbb{K})$ still corresponds to an R-parabolic subgroup $P_{\lambda}$ and an R-Levi subgroup $L_{\lambda}$ of $G$ (see [Bate et al. 2012, Section 2.2] for the case $\mathbb{K}=\mathbb{R}$ ). If $H$ is a reductive subgroup of $G$ then we write $Y(H, \mathbb{Q})$, etc., to denote the above constructions for $H$ instead of $G$.

2D. G-varieties and cocharacter-closure. We recall the following fundamental definition from [Bate et al. 2015, Definition 1.2], which extends the one given in [Bate et al. 2013, Definition 3.8].

Definition 2.3. A subset $S$ of $V$ is said to be cocharacter-closed over $k$ (for $G$ ) if for every $v \in S$ and $\lambda \in Y_{k}$ such that $v^{\prime}:=\lim _{a \rightarrow 0} \lambda(a) \cdot v$ exists, we have $v^{\prime} \in S$.

This notion is explored in detail in [Bate et al. 2015]. In this section, we content ourselves with collecting some results from that paper, together with the earlier paper [Bate et al. 2013]. These results, most of which are also needed in the sequel, give a flavour of what is known about the notion of cocharacter-closure in the case that the subset involved is a single $G(k)$-orbit. 
Remark 2.4. The geometric orbit $G \cdot v$ is Zariski-closed if and only if it is cocharacter-closed over $\bar{k}$, by the Hilbert-Mumford Theorem [Kempf 1978, Theorem 1.4].

Theorem 2.5 [Bate et al. 2015, Corollary 5.1]. Suppose $v \in V$ is such that $G(k) \cdot v$ is cocharacter-closed over $k$. Then whenever $v^{\prime}=\lim _{a \rightarrow 0} \lambda(a) \cdot v$ exists for some $\lambda \in Y_{k}$, there exists $u \in R_{u}\left(P_{\lambda}\right)(k)$ such that $v^{\prime}=u \cdot v$.

Proposition 2.6 [Bate et al. 2015, Proposition 5.5]. Let $v \in V$ such that $G_{v}\left(k_{s}\right)$ is $\Gamma$-stable and let $k^{\prime} / k$ be a separable algebraic extension. If $G\left(k^{\prime}\right) \cdot v$ is cocharacterclosed over $k^{\prime}$, then $G(k) \cdot v$ is cocharacter-closed over $k$.

Theorem 2.7 [Bate et al. 2015, Theorem 5.4]. Suppose $S$ is a k-defined torus of $G_{v}$ and set $L=C_{G}(S)$.

(i) If $G(k) \cdot v$ is cocharacter-closed over $k$, then $L(k) \cdot v$ is cocharacter-closed over $k$.

(ii) If $S$ is $k$-split, then $G(k) \cdot v$ is cocharacter-closed over $k$ if and only if $L(k) \cdot v$ is cocharacter-closed over $k$.

We note that, as described in the introduction, one of the main points of this paper is to show that the converse of Proposition 2.6 holds under certain extra hypotheses, and that the hypothesis of splitness can be removed in Theorem 2.7(ii) under the same hypotheses; see also [Bate et al. 2015, Theorem 1.5].

Our final result, a strengthening of Lemma 5.6 of [Bate et al. 2015], follows from the arguments given in the proof of that lemma.

Lemma 2.8. Let $V$ be an affine $G$-variety over $k$ and let $v \in V(k)$. Suppose there exists $\lambda \in Y_{k_{s}}$ such that $\lambda$ properly destabilizes $v$. Then there exists $\mu \in Y_{k}$ such that $v^{\prime}=\lim _{a \rightarrow 0} \mu(a) \cdot v$ exists, $v^{\prime}$ is not $G\left(k_{s}\right)$-conjugate to $v$ and $G_{v}\left(k_{s}\right)$ normalises $P_{\mu}$. In particular, $G(k) \cdot v$ is not cocharacter-closed over $k$.

Remark 2.9. The hypotheses of Lemma 2.8 are satisfied if $\lambda \in Y_{k_{s}}\left(Z\left(G^{0}\right)\right)$ destabilizes $v$ but does not fix $v$. For if $v^{\prime}:=\lim _{a \rightarrow 0} \lambda(a) \cdot v$ is $G$-conjugate to $v$ then $v^{\prime}$ is $R_{u}\left(P_{\lambda}\right)$-conjugate to $v$ [Bate et al. 2013, Theorem 3.3]; but $R_{u}\left(P_{\lambda}\right)=1$, so this cannot happen.

\section{Spherical buildings and Tits' centre conjecture}

The simplicial building $\Delta_{k}$ of a semisimple algebraic group $G$ over $k$ is a simplicial complex, the simplices of which correspond to the $k$-defined parabolic subgroups of $G$ ordered by reverse inclusion. See [Tits 1974, §5] for a detailed description. Our aim in this section is to construct for an arbitrary reductive group $G$ over $k$, objects $\Delta_{k}(\mathbb{K})$ for $\mathbb{K}=\mathbb{R}$ or $\mathbb{Q}$ that correspond to the geometric realisation of the spherical building of $G^{0}$ over $k$ (or the set of $\mathbb{Q}$-points thereof) when $G^{0}$ is 
semisimple. These are slightly more general objects (possibly with a contribution from $Z\left(G^{0}\right)$ ) when $G^{0}$ is reductive. Recall that $\Gamma$ denotes the Galois group of $k_{s} / k$. Most of the notation and terminology below is developed in full detail in the paper [Bate et al. 2012] — we point the reader in particular to the constructions in [Bate et al. 2012, Sections 2, 6.3, 6.4]. For the purposes of this paper, we need to extend some of the results in [loc. cit.] (for example by incorporating the effect of the Galois group $\Gamma$ ), but rather than reiterating all the details, we just gather enough material to make our exposition here coherent.

3A. Definition of $\boldsymbol{\Delta}_{\boldsymbol{k}}(\mathbb{K})$. We first form the vector building $V_{k}(\mathbb{K} \ll)$ by identifying $\lambda$ in $Y_{k}(\mathbb{K})$ with $u \cdot \lambda$ for every $u \in R_{u}\left(P_{\lambda}\right)(k)$. The norm function on $Y_{k}(\mathbb{K})$ descends to $V_{k}(\mathbb{K})$, because it is $G$-invariant. This gives a well-defined function on $V_{k}(\mathbb{K})$, which we also call a norm, and makes $V_{k}(\mathbb{K})$ into a metric space.

Definition 3.1. (i) Define $\Delta_{k}(\mathbb{R})$ to be the unit sphere in $V_{k}(\mathbb{R})$ and $\Delta_{k}(\mathbb{Q})$ to be the projection of $V_{k}(\mathbb{Q}) \backslash\{0\}$ onto $\Delta_{k}(\mathbb{R})$.

(ii) Two points of $\Delta_{k}(\mathbb{K})$ are called opposite if they are antipodal on the sphere $\Delta_{k}(\mathbb{R})$.

(iii) It is clear that the conjugation action of $G(k)$ on $Y_{k}$ gives rise to an action of $G(k)$ on $\Delta_{k}(\mathbb{K})$ by isometries, and there is a natural $G(k)$-equivariant, surjective map $\zeta: Y_{k}(\mathbb{K}) \backslash\{0\} \rightarrow \Delta_{k}(\mathbb{K})$.

(iv) The apartments of $\Delta_{k}(\mathbb{K})$ are the sets $\Delta_{k}(T, \mathbb{K}):=\zeta\left(Y_{k}(T, \mathbb{K})\right)$ where $T$ runs over the maximal $k$-split tori of $G$.

(v) The metric space $\Delta_{k}(\mathbb{K})$ and its apartments have a simplicial structure, because any point $x=\zeta(\lambda)$ of $\Delta_{k}(\mathbb{K})$ gives rise to a $k$-defined parabolic subgroup $P_{\lambda}$ of $G^{0}$ (see Section 2C); the simplicial complex consists of the proper $k$-defined parabolic subgroups of $G^{0}$, ordered by reverse inclusion. We write $\Delta_{k}$ for the spherical building of $G$ over $k$ regarded purely as a simplicial complex. The simplicial spherical buildings of $G^{0}$ and of $\left[G^{0}, G^{0}\right]$ are the same. Our notion of opposite is compatible with the usual one for parabolic subgroups: if $\lambda \in Y(G)$ then $P_{-\lambda}$ is an opposite parabolic to $P_{\lambda}$.

To avoid tying ourselves in knots, when the distinction is not important to the discussion at hand, we loosely refer to either of the objects $\Delta_{k}(\mathbb{Q})$ and $\Delta_{k}(\mathbb{R})$ as the building of $G$ over $k$.

One can make analogous definitions of objects $\Delta_{k_{s}}(\mathbb{K})$ and $\Delta(\mathbb{K})=\Delta_{\bar{k}}(\mathbb{K})$ over $k_{s}$ and $\bar{k}$, respectively, with corresponding systems of apartments and maps $\zeta$. We write $\Delta_{k_{s}}$ and $\Delta$ for these spherical buildings regarded as simplicial complexes.

Because we are interested in rationality results, we need to know the relationship between $\Delta_{k}(\mathbb{K})$ and $\Delta_{k_{s}}(\mathbb{K})$. Given a $k$-defined reductive subgroup $H$ of $G$, we also want to relate $\Delta_{k}(H, \mathbb{K})$ to $\Delta_{k}(\mathbb{K})$, where $\Delta_{k}(H, \mathbb{K})$ denotes the building of 
$H$ over $k$. It is easy to see that the $\Gamma$-action on cocharacters descends (via $\zeta$ ) to $\Gamma$-actions by isometries on $\Delta_{k_{s}}(\mathbb{K})$ and $\Delta(\mathbb{K})$.

Lemma 3.2. (i) There are naturally occurring copies of $\Delta_{k}(\mathbb{K})$ inside $\Delta_{k_{s}}(\mathbb{K})$ and $\Delta(\mathbb{K})$. We can in fact identify $\Delta_{k}(\mathbb{K})$ with the set of $\Gamma$-fixed points of $\Delta_{k_{s}}(\mathbb{K})$.

(ii) Let $H$ be a $k$-defined reductive subgroup of $G$. Then there is a naturally occurring copy of $\Delta_{k}(H, \mathbb{K})$ inside $\Delta_{k}(\mathbb{K})$.

Proof. (i) It is clear that $Y_{k}(\mathbb{K}) \subseteq Y_{k_{s}}(\mathbb{K}) \subseteq Y(\mathbb{K})$, and $Y_{k}(\mathbb{K})$ is precisely the set of $\Gamma$-fixed points in $Y_{k_{s}}(\mathbb{K})$. Since $R_{u}\left(P_{\lambda}\right)(k)$ acts simply transitively on the set of $k$-defined R-Levi subgroups of $P_{\lambda}$, two $k$-defined cocharacters $\lambda$ and $\mu$ are $R_{u}\left(P_{\lambda}\right)$-conjugate if and only if they are $R_{u}\left(P_{\lambda}\right)\left(k_{s}\right)$-conjugate if and only if they are $R_{u}\left(P_{\lambda}\right)(k)$-conjugate. Following this observation through the definition of $\Delta_{k}(\mathbb{K})$, $\Delta_{k_{s}}(\mathbb{K})$ and $\Delta(\mathbb{K})$ is enough to prove the first assertion of (i). It is clear that $\Delta_{k}(\mathbb{K})$ is fixed by $\Gamma$. Conversely, let $x \in \Delta_{k_{s}}(\mathbb{K})$ be fixed by $\Gamma$. Let $P$ be the parabolic subgroup associated to $x$. Then $P$ is $k_{s}$-defined and $\Gamma$-stable, so $P$ is $k$-defined. Pick a $k$-defined maximal torus $T$ of $P$. There exists $\lambda \in Y_{k_{s}}(T, \mathbb{K})$ such that $P=P_{\lambda}$ [Springer 1998, 8.4.4, 8.4.5]. Each $\gamma \in \Gamma$ maps $\lambda$ to a $R_{u}(P)\left(k_{s}\right)$-conjugate of $\lambda$. Now $R_{u}(P)$ acts simply transitively on the set of Levi subgroups of $P$, and each maximal torus of $P$ is contained in a unique Levi subgroup [Springer 1998, 8.4.4], so $R_{u}(P)$ acts freely on the set of maximal tori of $P$. But $T$ is $\Gamma$-stable, so we must have that $\Gamma$ fixes $\lambda$. Hence $x \in \Delta_{k}(\mathbb{K})$, as required.

(ii) In analogy with the first assertion of (i) (although it is slightly more subtle), the key observation is that if $\lambda, \mu \in Y_{k}(H)$ are $R_{u}\left(P_{\lambda}(G)\right)(k)$-conjugate, then they are in fact $R_{u}\left(P_{\lambda}(H)\right)(k)$-conjugate (see [Bate et al. 2011, Lemma 3.3(i)]). Observe also that the restriction of a $\Gamma$ - and $G$-invariant norm on $Y$ to $Y(H)$ gives a $\Gamma$ - and $H$-invariant norm on $Y(H)$.

Henceforth, we write $\Delta_{k}(\mathbb{K}) \subseteq \Delta_{k_{s}}(\mathbb{K}) \subseteq \Delta(\mathbb{K})$ and $\Delta_{k}(H, \mathbb{K}) \subseteq \Delta_{k}(\mathbb{K})$ without any further comment. One note of caution: the inclusion $\Delta_{k}(H, \mathbb{K}) \subseteq \Delta_{k}(\mathbb{K})$ does not in general respect the simplicial structures on these objects.

3B. Convex subsets. Because any two parabolic subgroups of $G$ contain a common maximal torus, any two points $x, y \in \Delta(\mathbb{K})$ are contained in a common apartment and, as long as these points are not opposite each other, there is a unique geodesic $[x, y]$ joining them. This geodesic does not depend on the apartment we find containing $x$ and $y$; in particular, this can be done inside $\Delta_{k}(\mathbb{K})$ if $x, y \in \Delta_{k}(\mathbb{K})$ and inside $\Delta_{k}(H)$ if $x, y \in \Delta_{k}(H)$ for some reductive subgroup $H$ of $G$. This leads to the following key definitions: 
Definition 3.3. (i) A subset $\Sigma \subseteq \Delta(\mathbb{K})$ is called convex if whenever $x, y \in \Sigma$ are not opposite then $[x, y] \subseteq \Sigma$. It follows from the discussion above that $\Delta_{k}(\mathbb{K})$ is a convex subset of $\Delta(\mathbb{K})$.

(ii) Given a convex subset $\Sigma$ of $\Delta(\mathbb{K})$, its preimage $C:=\zeta^{-1}(\Sigma) \cup\{0\}$ in $Y(\mathbb{K})$ is a union of cones $C_{T}:=C \cap Y(T, \mathbb{K})$, where $T$ runs over the maximal tori of $G$. The subset $\Sigma$ is called polyhedral if each $C_{T}$ is a polyhedral cone and $\Sigma$ is said to have finite type if the set of cones $\left\{g \cdot C_{g^{-1}} T g \mid g \in G\right\}$ is finite for all $T$.

(iii) A convex subset $\Sigma$ of $\Delta(\mathbb{K})$ is called a subcomplex if it is a union of simplices (that is, if $\lambda, \mu \in Y(\mathbb{K})$ are such that $P_{\lambda}=P_{\mu}$, then $\zeta(\lambda) \in \Sigma$ if and only if $\zeta(\mu) \in \Sigma$ ) and if that union of simplices forms a subcomplex in the simplicial building $\Delta$. In such a circumstance, we denote the subcomplex of $\Delta$ arising in this way by $\Sigma$ also; note that $\Sigma$ is convex in the sense of part (i) above if and only if $\Sigma$ - regarded as a subcomplex of the simplicial building — is convex in the sense of simplicial buildings.

The definitions above have obvious analogues for the buildings $\Delta_{k}(\mathbb{K})$ and $\Delta_{k_{s}}(\mathbb{K})$.

There is an addition operation on the set $V(\mathbb{K})$, given as follows. Let $\varphi: Y(\mathbb{K}) \rightarrow$ $V(\mathbb{K})$ be the canonical projection. Choose a maximal torus $T$ of $G$ and $\lambda, \mu \in$ $Y(T, \mathbb{K})$ such that $\varphi(\lambda)=x$ and $\varphi(\mu)=y$; we define $x+y \in V(\mathbb{K})$ by $x+y=$ $\varphi(\lambda+\mu)$. It can be shown that this does not depend on the choice of $T$; moreover, for any $g \in G, g \cdot(x+y)=g \cdot x+g \cdot y$.

3C. The destabilizing locus and complete reducibility. For this paper, a particularly important class of convex subsets arises from $G$-actions on affine varieties. Given an affine $G$-variety $V$ and a point $v \in V$, set

$$
\Sigma_{v}:=\left\{\zeta(\lambda) \mid \lambda \in Y \text { and } \lim _{a \rightarrow 0} \lambda(a) \cdot v \text { exists }\right\} \subseteq \Delta(\mathbb{Q}) .
$$

We call this subset the destabilizing locus for $v$; it is a convex subset of $\Delta(\mathbb{Q})$ by [Bate et al. 2012, Lemma 5.5] (note that $\Sigma_{v}$ coincides with $E_{V,\{v\}}(\mathbb{Q})$ in the language of [Bate et al. 2012]). Similarly we write $\Sigma_{v, k}$ (resp. $\Sigma_{v, k_{s}}$ ) for the image in $\Delta_{k}(\mathbb{Q})\left(\right.$ resp. $\left.\Delta_{k_{s}}(\mathbb{Q})\right)$ of the $k$-defined (resp. $k_{s}$-defined) characters destabilizing $v$. If $H$ is a reductive subgroup of $G$, then we write $\Sigma_{v, k}(H)$ for the destabilizing locus for $v$ with respect to $H$.

Definition 3.4. A subset $\Sigma$ of $\Delta(\mathbb{K})$ is called completely reducible if every point of $\Sigma$ has an opposite in $\Sigma$.

Lemma 3.5. Let $v \in V$. Then:

(i) Given $\lambda \in Y_{k}$ such that $\zeta(\lambda) \in \Sigma_{v, k}$, $\lambda$ has an opposite in $\Sigma_{v, k}$ if and only if there exists $u \in R_{u}\left(P_{\lambda}\right)(k)$ such that $u \cdot \lambda$ fixes $v$, if and only if there exists $u \in R_{u}\left(P_{\lambda}\right)(k)$ such that $\lim _{a \rightarrow 0} \lambda(a) \cdot v=u^{-1} \cdot v$. 
(ii) The subset $\Sigma_{v, k}$ is completely reducible if and only if $G(k) \cdot v$ is cocharacterclosed over $k$.

(iii) The subset $\Sigma_{v}$ is completely reducible if and only if the orbit $G \cdot v$ is closed in $V$.

Proof. We have that $\Sigma_{v}$ (resp. $\Sigma_{v, k}$ ) is completely reducible if and only if for every $\lambda \in Y$ (resp. $\lambda \in Y_{k}$ ) such that $\lim _{a \rightarrow 0} \lambda(a) \cdot v$ exists, there is some $u \in R_{u}\left(P_{\lambda}\right)$ (resp. $u \in R_{u}\left(P_{\lambda}\right)(k)$ ) such that both $u \cdot \lambda$ and $-(u \cdot \lambda)$ destabilize $v$. But this is true if and only if $u \cdot \lambda$ fixes $v$, which is equivalent to the fact that $\lim _{a \rightarrow 0} \lambda(a) \cdot v=u^{-1} \cdot v$, by [Bate et al. 2013, Lemma 2.12]. This gives part (i). Part (ii) now follows from Theorem 2.5, and part (iii) from Remark 2.4.

3D. The strong centre conjecture and quasi-states. The aim of the paper [Bate et al. 2012] is to study a strengthened version of Tits' centre conjecture for $\Delta_{k_{s}}(\mathbb{K})$. Let $\mathcal{G}$ denote the group of transformations of $\Delta_{k_{s}}(\mathbb{K})$ generated by the isometries arising from the action of $G\left(k_{s}\right)$ and the action of $\Gamma$. Note that elements of $\mathcal{G}$ map $k_{s}$-defined parabolic subgroups of $G$ to $k_{s}$-defined parabolic subgroups of $G$, so they give rise to automorphisms of the simplicial building $\Delta_{k_{s}}$. Given a convex subset $\Sigma$ of $\Delta_{k_{s}}(\mathbb{K})$, we call a point $x \in \Sigma$ a $\mathcal{G}$-centre if it is fixed by all the elements of $\mathcal{G}$ that stabilize $\Sigma$ setwise. We can now formulate the original centre conjecture in our setting.

Theorem 3.6. Suppose $\Sigma \subseteq \Delta_{k_{s}}(\mathbb{K})$ is a convex non-completely reducible subcomplex. Then $\Sigma$ has a $\mathcal{G}$-centre.

Proof. Theorem 1.2 asserts the existence of a stable simplex in the subcomplex (note that the simplicial structure on $\Delta(\mathbb{K})$ does not "see" the difference between $G$ and $G^{0}$, or between $G^{0}$ and its semisimple part, so the proof of the centre conjecture for subcomplexes of spherical buildings still works for the more general class of objects we have described). Now any element of $\mathcal{G}$ that fixes a simplex also fixes its barycentre (because the action is via isometries), and we are done.

In the strong centre conjecture [Bate et al. 2012, Conjecture 2.10], one replaces convex non-completely reducible subcomplexes with convex non-completely reducible subsets. Most of [loc. cit.] deals with the special case when $k=\bar{k}$ and considers only the isometries of $\Delta_{k}(\mathbb{K})$ coming from the action of $G$. We need to take the action of $\Gamma$ into account, so we briefly indicate some of the key changes that must be made to the constructions in [loc. cit.] in order to make the results go through; see also the comments in [loc. cit., Section 6.3].

Definition 3.7. We recall the notion of a $\mathbb{K}$-quasi-state $\Xi$ from [Bate et al. 2012, Definition 3.1]: this is an assignment of a finite set of characters $\Xi(T)$ to each 
maximal torus $T$ of $G$ satisfying certain conditions (see [loc. cit.] for a precise statement).

The groups $G$ and $\Gamma$ act on quasi-states: given a $\mathbb{K}$-quasi-state $\Xi$ and $g \in G$ and $\gamma \in \Gamma$ we define new quasi-states $g_{*} \Xi$ and $\gamma_{*} \Xi$ by

$$
g_{*} \Xi(T):=g_{!} \Xi\left(g^{-1} T g\right), \quad \gamma_{*} \Xi(T):=\gamma_{!}\left(\gamma^{-1} \cdot T\right),
$$

where for a character $\chi$ of a torus $T, g_{!} \chi$ is a character of the torus $g T g^{-1}$ given by $\left(g_{!} \chi\right)\left(\mathrm{gtg}^{-1}\right):=\chi(t)$ for all $t \in T$, and similarly $\gamma_{!} \chi$ is a character of $\gamma \cdot T$ given by $\left(\gamma_{!} \chi\right)(\gamma \cdot t):=\chi(t)$ for all $t \in T$.

We say a quasi-state is defined over a field $k^{\prime}$ if it assigns $k^{\prime}$-defined characters to $k^{\prime}$-defined maximal tori. Recall also the notions of boundedness, admissibility and quasi-admissibility for $\mathbb{K}$-quasi-states, [Bate et al. 2012, Definitions 3.1 and 3.2].

With these definitions in hand, we can extend [loc. cit., Lemma 3.8] as follows:

Lemma 3.8. Let $\Upsilon$ be a $\mathbb{K}$-quasi-state which is defined over $k_{s}$ and define $\Xi:=$ $\bigcup_{\gamma \in \Gamma} \gamma_{*} \Upsilon$ by $\Xi(T):=\bigcup_{\gamma \in \Gamma}\left(\gamma_{*} \Upsilon\right)(T)$ for each maximal torus $T$ of $G$. Then $\Xi$ is $a \mathbb{K}$-quasi-state which is defined over $k_{s}$, and it is bounded (resp. quasi-admissible, admissible at $\lambda$ ) if $\Upsilon$ is. Moreover, by construction, $\Xi$ is $\Gamma$-stable.

Proof. There are two points which need to be made in order for the arguments already in the proof of [Bate et al. 2012, Lemma 3.8] to go through. First note that given a $k_{s}$-defined maximal torus $T$ of $G$ the set of Galois conjugates of $T$ is finite (because $T$ is defined over some finite extension of $k$ ). This means that, because $\Upsilon$ is $k_{s}$-defined, $\Xi(T)$ is still finite, so $\Xi$ is a $\mathbb{K}$-quasi-state. Now, for boundedness we need to check that if $\Upsilon$ is bounded then the set $\bigcup_{\gamma \in \Gamma}\left(\bigcup_{g \in G} g_{*}\left(\gamma_{*} \Upsilon\right)(T)\right)$ is finite for some (and hence all) $k_{s}$-defined maximal tori $T$ of $G$. Since we can choose any $k_{s}$-defined maximal torus $T$, we choose one that is actually $k$-defined, and then

$$
\begin{aligned}
g_{*}\left(\gamma_{*} \Upsilon\right)(T) & =g_{!}\left(\gamma_{*} \Upsilon\right)\left(g^{-1} T g\right)=g_{!}\left(\gamma_{!} \Upsilon\left(\gamma^{-1} \cdot\left(g^{-1} T g\right)\right)\right) \\
& =g_{!}\left(\gamma_{!} \Upsilon\left(\left(\gamma^{-1} \cdot g\right)^{-1} T\left(\gamma^{-1} \cdot g\right)\right)\right) \\
& =\gamma_{!}\left(\gamma^{-1} \cdot g\right)_{!} \Upsilon\left(\left(\gamma^{-1} \cdot g\right)^{-1} T\left(\gamma^{-1} \cdot g\right)\right)=\gamma_{!}\left(\left(\gamma^{-1} \cdot g\right)_{*} \Upsilon\right)(T) .
\end{aligned}
$$

Therefore, we can write

$$
\bigcup_{\gamma \in \Gamma}\left(\bigcup_{g \in G}\left(g_{*}\left(\gamma_{*} \Upsilon\right)\right)(T)\right)=\bigcup_{\gamma \in \Gamma} \gamma_{!}\left(\bigcup_{g \in G}\left(\gamma^{-1} \cdot g\right)_{*} \Upsilon(T)\right) .
$$

Now, since $\Upsilon$ is bounded, the second union on the RHS is finite for every $\gamma$, and because $\Upsilon$ is $k_{s}$-defined and the set of Galois conjugates of a $k_{s}$-defined character is finite, the whole RHS is finite. This proves the boundedness assertion. The other assertions follow as in [loc. cit.] 
Using Lemma 3.8 we can ensure that, when appropriate, the states and quasistates constructed during the course of the paper [Bate et al. 2012] are Galois-stable. In particular, we get the following variant of [loc. cit., Theorem 5.5]:

Theorem 3.9. Suppose $\Sigma \subseteq \Delta(\mathbb{Q})$ is a convex polyhedral non-completely reducible subset of finite type contained in a single apartment of $\Delta(\mathbb{Q})$. Then $\Sigma$ has a $\mathcal{G}$-centre. In particular, if $\Sigma$ is stabilized by all of $\Gamma$, then there exists $a \Gamma$-fixed point in $\Sigma$.

Proof. Using Lemma 3.8, one can ensure that the quasi-state constructed in [Bate et al. 2012, Lemma 5.2] which is used in the proof of [loc. cit., Theorem 5.5] is also stable under the relevant elements of $\mathcal{G}$. The proofs in [loc. cit.] now go through.

Remark 3.10. In our application of Theorem 3.9 to the proof of Theorem 1.3 below, $\Sigma$ is a $\Gamma$-stable subset of $\Delta_{k_{s}}(\mathbb{Q})$ and we want to show that $\Sigma$ has a $\Gamma$-fixed point. A striking feature of Theorem 3.9 is that we do not require the apartment containing $\Sigma$ to be $k_{s}$-defined: it can be any apartment of the building $\Delta(\mathbb{K})=\Delta_{\bar{k}}(\mathbb{K})$.

We note here that, unfortunately, we do not know a priori that any cocharacter corresponding to the fixed point given by Theorem 3.9 properly destabilizes $v$. Moreover, we may need to consider cocharacters of $Z\left(G^{0}\right)$, which do not correspond to simplices of the spherical building at all. These technical issues are at the heart of many of the complications in the proofs in Section 4 below.

\section{Proofs of the main results}

Having put in place the building-theoretic technology needed for our proofs, we start this section with a few more technical results to be used for the main theorems. As always, $V$ denotes a $k$-defined affine $G$-variety, and $v \in V$. One obstacle to proving Theorems 1.1 and 1.3 is that we need to deal with cocharacters that live in $Z\left(G^{0}\right)$, which are not detected by the simplicial building (cf. the proof of Theorem 3.6). An extra problem for Theorem 1.3 is that we need to factor out some simple components of $G^{0}$. The following results let us deal with these difficulties.

Let $N$ be a product of certain simple factors of $G^{0}$, and let $S$ be a torus of $Z\left(G^{0}\right)$. Let $M$ be the product of the remaining simple factors of $G^{0}$ together with $Z\left(G^{0}\right)$. Suppose that $N$ and $S$ are normal in $G$ (this implies that $M$ is normal in $G$ as well), and that $N$ and $S$ both fix $v$. Set $G_{1}=G / N S$ and let $\pi: G \rightarrow G_{1}$ be the canonical projection. Since $Z\left(G^{0}\right)$ is $k_{s}$-defined and $k_{s}$-split, $S$ is $k_{s}$-defined, and it is clear that $N$ is $k_{s}$-defined. So $G_{1}$ and $\pi$ are $k_{s}$-defined. We have a $k_{s}$-defined action of $G_{1}$ on the fixed point set $V^{N S}$ (note that $V^{N S}$ is $G$-stable).

Lemma 4.1. (i) For any $\mu_{1} \in Y_{k_{s}}\left(G_{1}\right)$, there exist $n \in \mathbb{N}$ and $\mu \in Y_{k_{s}}(M)$ such that $\pi \circ \mu=n \mu_{1}$.

(ii) Let $\lambda \in Y_{k_{s}}$. Then $\lambda$ destabilizes $v$ over $k_{s}$ in $V$ if and only if $\pi \circ \lambda$ destabilizes $v$ over $k_{s}$ in $V^{N S}$. Moreover, if this is the case then $\lim _{a \rightarrow 0} \lambda(a) \cdot v=$ 
$\lim _{a \rightarrow 0}(\pi \circ \lambda)(a) \cdot v$ belongs to $R_{u}\left(P_{\lambda}(G)\right)\left(k_{s}\right) \cdot v$ if and only if it belongs to $R_{u}\left(P_{\pi \circ \lambda}\left(G_{1}\right)\right)\left(k_{s}\right) \cdot v$.

(iii) $G_{1}\left(k_{s}\right) \cdot v$ is cocharacter-closed over $k_{s}$ if and only if $G\left(k_{s}\right) \cdot v$ is cocharacterclosed over $k_{s}$.

Proof. (i) Let $\mu_{1} \in Y_{k_{s}}\left(G_{1}\right)$. Since $\mu_{1}$ is $k_{s}$-defined, we can choose a $k_{s}$-defined maximal torus $T_{1} \subseteq G_{1}$ with $\mu_{1} \in Y_{k_{s}}\left(T_{1}\right)$. Since $\pi$ is separable and $k_{s}$-defined, $\pi^{-1}\left(T_{1}\right) \subseteq G$ is $k_{s}$-defined [Springer 1998, Corollary 11.2.14]. Hence $\pi^{-1}\left(T_{1}\right)$ has a $k_{s}$-defined maximal torus $T$. Let $T^{\prime}=T \cap M$, a $\bar{k}$-defined torus of $M$. Now $\pi\left(T^{\prime}\right)=\pi(T)$ is a maximal torus of $G_{1}$ by [Borel 1991, Proposition 11.14(1)]; but $\pi\left(T^{\prime}\right)$ is contained in $T_{1}$, so we must have $\pi\left(T^{\prime}\right)=T_{1}$. The surjection $T^{\prime} \rightarrow T_{1}$ induces a surjection $\mathbb{Q} \otimes_{\mathbb{Z}} Y_{\bar{k}}\left(T^{\prime}\right) \rightarrow \mathbb{Q} \otimes_{\mathbb{Z}} Y_{\bar{k}}\left(T_{1}\right)$ (the map before tensoring maps onto a finite-index subgroup: e.g., by transposing the injective map on character groups [Waterhouse 1979, Theorem 7.3]), hence there exist $n \in \mathbb{N}$ and $\mu \in Y_{\bar{k}}\left(T^{\prime}\right)$ such that $\pi \circ \mu=n \mu_{1}$. As $\mu \in Y_{\bar{k}}(T)=Y_{k_{s}}(T), \mu$ is $k_{s}$-defined as required.

(ii) The first assertion is immediate, as is the assertion that the limits coincide. Since $\pi$ is an epimorphism, we have $\pi\left(R_{u}\left(P_{\lambda}(G)\right)\right)=R_{u}\left(P_{\pi \circ \lambda}\left(G_{1}\right)\right)$ (see [Conrad et al. 2010, Corollary 2.1.9]). Moreover, since $\lambda$ normalises $N S$, the restriction of $\pi$ to $R_{u}\left(P_{\lambda}(G)\right)$ is separable (see [Conrad et al. 2010, Proposition 2.1.8(3) and Remark 2.1.11]) and $k_{s}$-defined, and hence is surjective on $k_{s}$-points (cf. [Waterhouse 1979, Corollary 18.5]). This implies that if the common limit $v^{\prime}$ is in $R_{u}\left(P_{\pi \circ \lambda}\left(G_{1}\right)\right)\left(k_{s}\right) \cdot v$, it is contained in $R_{u}\left(P_{\lambda}(G)\right)\left(k_{s}\right) \cdot v$. The reverse implication is clear, since $\pi$ is $k_{s}$-defined.

(iii) Suppose $G\left(k_{s}\right) \cdot v$ is not cocharacter-closed over $k_{s}$. Then there exists $\lambda \in Y_{k_{s}}$ such that $\lim _{a \rightarrow 0} \lambda(a) \cdot v$ exists but does not belong to $R_{u}\left(P_{\lambda}(G)\right)\left(k_{s}\right) \cdot v$. Then $\lim _{a \rightarrow 0}(\pi \circ \lambda)(a) \cdot v$ exists but does not belong to $R_{u}\left(P_{\lambda}\left(G_{1}\right)\right)\left(k_{s}\right) \cdot v$, by part (ii). Hence $G_{1}\left(k_{s}\right) \cdot v$ is not cocharacter-closed over $k_{s}$, by Theorem 2.5.

Now suppose $G_{1}\left(k_{s}\right) \cdot v$ is not cocharacter-closed over $k_{s}$. Then there exists $\mu_{1} \in Y_{k_{s}}\left(G_{1}\right)$ such that $v^{\prime}:=\lim _{a \rightarrow 0} \mu_{1}(a) \cdot v$ exists and does not belong to $R_{u}\left(P_{\mu}\left(G_{1}\right)\right)\left(k_{s}\right) \cdot v$. Replacing $\mu_{1}$ with a positive multiple $n \mu_{1}$ of $\mu_{1}$ if necessary, it follows from part (i) that there exists $\mu \in Y_{k_{s}}$ such that $\pi \circ \mu=\mu_{1}$. Then $\lim _{a \rightarrow 0} \mu(a) \cdot v$ is equal to $v^{\prime}$ and $v^{\prime}$ does not belong to $R_{u}\left(P_{\lambda}(G)\right)\left(k_{s}\right) \cdot v$, by part (ii). Hence $G\left(k_{s}\right) \cdot v$ is not cocharacter-closed over $k_{s}$, by Theorem 2.5.

Remark 4.2. We insist in Lemma 4.1(i) that $\lambda$ be a cocharacter of $M$ because we need this in the proof of Theorem 1.3.

Lemma 4.3. Let $G$ be connected, let $S$ be a $k_{s}$-torus of $G_{v}$ and set $L=C_{G}(S)$. Suppose that for every $\lambda, \mu \in Y_{k_{s}}$ such that $P_{\lambda}=P_{\mu}$, either both of $\lambda$ and $\mu$ destabilize $v$ or neither does. Then for every $\lambda, \mu \in Y_{k_{s}}(L)$ such that $P_{\lambda}(L)=P_{\mu}(L)$, either both of $\lambda$ and $\mu$ destabilize $v$ or neither does. 
Proof. Let $\lambda, \mu \in Y_{k}(L)$ such that $P_{\lambda}(L)=P_{\mu}(L)$. We can choose $\sigma \in Y_{k}(S)$ such that $L_{\sigma}=L$ [Bate et al. 2015, Lemma 2.5]. Then $P_{\lambda}\left(L_{\sigma}\right)=P_{\mu}\left(L_{\sigma}\right)$ and there exists $n \in \mathbb{N}$ such that $P_{n \sigma+\lambda}=P_{\lambda}\left(L_{\sigma}\right) R_{u}\left(P_{\sigma}\right)$ and $P_{n \sigma+\mu}=P_{\mu}\left(L_{\sigma}\right) R_{u}\left(P_{\sigma}\right)$ [Bate et al. 2005, Lemma 6.2(i)]. By hypothesis, either both of $n \sigma+\lambda$ and $n \sigma+\mu$ destabilize $v$, or neither one does. In the first case, since $\sigma$ fixes $v$, both $\lambda$ and $\mu$ must destabilize $v$. Conversely, in the second case neither $\lambda$ nor $\mu$ can destabilize $v$.

Lemma 4.4. Let $T$ be a maximal torus of $G$, let $\mu_{1}, \ldots, \mu_{r} \in Y(T) \backslash\{0\}$, let $\mu=$ $\sum_{i=1}^{r} \mu_{i}$ and assume $\mu \neq 0$. Suppose $g \in G$ and $g \cdot \zeta\left(\mu_{i}\right)=\zeta\left(\mu_{i}\right)$ for all $1 \leq i \leq r$. Then $g \cdot \zeta(\mu)=\zeta(\mu)$.

Proof. Clearly, there is a permutation $\tau \in S_{r}$ such that none of the sums $\sum_{i=1}^{t} \mu_{\tau(i)}$ is 0 for $1 \leq t \leq r$. Consider the special case $r=2$ (the general case follows easily by induction on $r$ ). Recall the addition operation + on $V(\mathbb{K})$ and the canonical projection $\varphi: Y(\mathbb{K}) \rightarrow V(\mathbb{K})$ from Section 3B. Let $\xi: V(\mathbb{K}) \backslash\{0\} \rightarrow \Delta(\mathbb{K})$ be the canonical projection. Note that $\varphi$ and $\xi$ are $G$-equivariant. Moreover, as $g$ fixes $\zeta\left(\mu_{1}\right)$ and $g$ acts as an isometry, $g$ fixes $\varphi\left(\mu_{1}\right)$, and likewise $g$ fixes $\varphi\left(\mu_{2}\right)$. We have

$$
\begin{aligned}
g \cdot \zeta(\mu) & =g \cdot \zeta\left(\mu_{1}+\mu_{2}\right)=g \cdot \xi\left(\varphi\left(\mu_{1}+\mu_{2}\right)\right)=\xi\left(g \cdot \varphi\left(\mu_{1}+\mu_{2}\right)\right) \\
& =\xi\left(g \cdot\left(\varphi\left(\mu_{1}\right)+\varphi\left(\mu_{2}\right)\right)\right)=\xi\left(g \cdot \varphi\left(\mu_{1}\right)+g \cdot \varphi\left(\mu_{2}\right)\right) \\
& =\xi\left(\varphi\left(\mu_{1}\right)+\varphi\left(\mu_{2}\right)\right)=\xi\left(\varphi\left(\mu_{1}+\mu_{2}\right)\right)=\zeta\left(\mu_{1}+\mu_{2}\right)=\zeta(\mu),
\end{aligned}
$$

as required.

We now have everything in place to prove Theorem 1.1.

Proof of Theorem 1.1. For part (i), suppose $v \in V(k)$ and $G\left(k_{s}\right) \cdot v$ is not cocharacterclosed over $k_{s}$. Clearly there is no harm in assuming $S$ is a maximal $k$-defined torus of $G_{v}$, so we shall do this. Since the closed subgroup $\overline{G_{v}\left(k_{s}\right)}$ generated by $G_{v}\left(k_{s}\right)$ is $k_{s}$-defined and $\Gamma$-stable, it is $k$-defined. Hence $S$ is a maximal torus of $\overline{G_{v}\left(k_{s}\right)}$; in particular, $S$ is a maximal $k_{s}$-defined torus of $G_{v}$. Set $H=C_{G}(S)$. If $\sigma \in Y_{k}(H)$ and $\sigma$ destabilizes $v$ but does not fix $v$ then $\sigma$ properly destabilizes $v$ over $k_{s}$ for $G$, by [Bate et al. 2015, Lemma 2.8]. Hence it is enough to prove that such a $\sigma$ exists.

By Theorem 2.7(ii), $H\left(k_{s}\right) \cdot v$ is not cocharacter-closed over $k_{s}$. So we can choose $\mu \in Y_{k_{s}}(H)$ such that $\mu$ properly destabilizes $v$ over $k_{s}$ for $H$. If $\mu \in Y_{k_{s}}\left(Z\left(H^{0}\right)\right)$ then we are done by Lemma 2.8 and Remark 2.9. So assume otherwise. Then $P_{\mu}\left(H^{0}\right)$ is a proper subgroup of $H^{0}$. By Lemma $4.3, \Sigma_{v, k_{s}}(H)$ is a subcomplex of $\Delta_{k_{s}}(H, \mathbb{K})$. It follows from Lemma 3.5 that $\Sigma_{v, k_{s}}(H)$ is not completely reducible, since if $Q$ is an opposite parabolic to $P_{\mu}\left(H^{0}\right)$ in $H^{0}$ then there exists $\mu^{\prime} \in Y_{k_{s}}(H)$ such that $P_{\mu^{\prime}}=Q$ and $\mu^{\prime}$ is opposite to $\mu$, which is impossible. Clearly, $\Sigma_{v, k_{s}}(H)$ is $\Gamma$ - and $H_{v}\left(k_{s}\right)$-stable, so by Theorem 3.6 there is a $\Gamma$ - and $H_{v}\left(k_{s}\right)$-fixed simplex 
$s \in \Sigma_{v, k_{s}(H)}$, corresponding to some proper parabolic subgroup $P$ of $H^{0}$. There exists $\sigma \in Y_{k}(H)$ such that $P=P_{\sigma}\left(H^{0}\right)$ [Bate et al. 2013, Lemma 2.5(ii)], and $\sigma$ destabilizes $v$ by construction. Now $\sigma \notin Y_{k_{s}}\left(Z\left(H^{0}\right)\right)$ since $P$ is proper. But every $k_{s}$-defined torus of $H_{v}\left(k_{s}\right)$ is contained in $Z\left(H^{0}\right)$ (since $S$ is contained in $Z\left(H^{0}\right)$ ), so $\sigma$ does not fix $v$. As $\sigma$ commutes with $S$, it follows from [Bate et al. 2015, Lemma 2.8] that $v^{\prime}:=\lim _{a \rightarrow 0} \sigma(a) \cdot v$ does not lie in $H\left(k_{s}\right) \cdot v$. This completes the proof of (i).

For part (ii), Proposition 2.6 shows that if $G\left(k^{\prime}\right) \cdot v$ is cocharacter-closed over $k^{\prime}$ then $G(k) \cdot v$ is cocharacter-closed over $k$. For the other direction, suppose that $G\left(k^{\prime}\right) \cdot v$ is not cocharacter-closed over $k^{\prime}$. Again by Proposition 2.6, we may assume $k^{\prime}=k_{s}$. Applying part (i) with $S=1$, we find $\sigma \in Y_{k}$ such that $\sigma$ properly destabilizes $v$ over $k_{s}$. In particular, $G(k) \cdot v$ is not cocharacter-closed over $k$. This finishes part (ii).

Part (iii) of Theorem 1.1 follows using similar arguments to those in the proof of [Bate et al. 2015, Theorem 5.7(ii)]. Let $S$ be a $k$-defined torus of $G_{v}$ and let $L=C_{G}(S)$. First, by the argument of [Bate et al. 2015, Lemma 6.2], we can assume without loss that $v \in V\left(k_{s}\right)$ without changing $\Sigma_{v, k_{s}}$. Second, by [Bate et al. 2015, Lemma 6.3] and the argument of the proof of [Bate et al. 2015, Theorem 6.1], we can pass to a suitable $G$-variety $W$ and find $w \in W(k)$ such that $\Sigma_{w, k_{s}}=\bigcap_{\gamma \in \Gamma} \gamma \cdot \Sigma_{v, k_{s}}$; in particular, $\Sigma_{w, k_{s}}$ is a subcomplex of $\Delta_{k}(\mathbb{Q})$ and $\Sigma_{w, k}=\Sigma_{v, k}$. The arguments of [Bate et al. 2015, Section 6] also show that $S$ fixes $w$. Hence we can assume without loss that $v \in V(k)$. As before, Lemma 4.3 implies that $\Sigma_{v, k_{s}}(L)$ is a subcomplex of $\Delta_{k_{s}}(L, \mathbb{K})$. We may thus apply part (ii) and assume $k=k_{s}$. But then $S$ is $k$-split, so the result follows from Theorem 2.7.

Remark 4.5. We do not know how to prove that $P_{\sigma}\left(G^{0}\right)$ from Theorem 1.1(i) is normalised by $G_{v}\left(k_{s}\right)$, but the proof does show that $P_{\sigma}\left(G^{0}\right)$ is normalised by $H_{v}\left(k_{s}\right)$.

Proof of Theorem 1.3. For part (i), suppose $v \in V(k)$ and $G\left(k_{s}\right) \cdot v$ is not cocharacterclosed over $k_{s}$. Recall that a connected algebraic group is nilpotent if and only if it contains just one maximal torus (see [Humphreys 1975, §21.4 Proposition B]). Let $G_{i}$ be a simple component of $G^{0}$. If $\operatorname{rank}\left(G_{i}\right)=1$ and $\operatorname{dim}\left(G_{i}\right)_{v} \geq 2$ then $\left(G_{i}\right)_{v}^{0}$ must contain a Borel subgroup $B_{i}$ of $G_{i}$ : but then the orbit map $G_{i} \rightarrow G_{i} \cdot v$ factors through the connected projective variety $G_{i} / B_{i}$ and hence is constant, so $\left(G_{i}\right)_{v}=G_{i}$.

Let $N$ be the product of the simple components of $G^{0}$ that fix $v$, and let $K$ be the product of the remaining simple components of $G^{0}$ together with $Z\left(G^{0}\right)^{0}$. Then $N$ and $K$ are $\Gamma$-stable, so they are $k$-defined. The next step is to factor out $N$ and reduce to the case when the stabilizer has nilpotent identity component. As in the proof of Theorem 1.1, $\overline{G_{v}\left(k_{s}\right)}$ is $k$-defined and we may assume $S$ is a maximal 
$k$-defined torus of $G_{v}$ and a $k_{s}$-defined maximal torus of $\overline{G_{v}\left(k_{s}\right)}$. We can choose $k$-defined tori $S_{0}$ of $K$ and $S_{2}$ of $N$ such that $S=S_{0} S_{2}$. Note that $K_{v}^{0}$ is nilpotentthis holds by assumption in case (a), and by the above argument in case (b) - so $S_{0}$ is the unique maximal $k$-defined torus of $\overline{K_{v}\left(k_{s}\right)}$.

Let $H_{0}=N_{G}\left(S_{0}\right)$, let $H=N_{H_{0}}(N)$ and let $M=C_{K}\left(S_{0}\right)$. Then $H_{0}$ is $k$-defined [Conrad et al. 2010, Lemma A.2.9], so $H$ is $k$-defined as it is $\Gamma$-stable and has finite index in $H^{0}$. Note that $H^{0}=N M=C_{G}\left(S_{0}\right)^{0}$, so $N$ is a product of simple components of $H^{0}$ and $M$ is the product of $Z\left(H^{0}\right)^{0}=S^{0} Z\left(G^{0}\right)^{0}$ with the remaining simple components of $H^{0}$. The subgroup $M$ of $H$ is normal, so it is $\Gamma$-stable and hence $k$-defined. Now $M_{v}^{0}$ is nilpotent since $K_{v}^{0}$ is, so $M_{v}^{0}$ has a unique maximal torus $S^{\prime}$-in particular, $S_{0} \subseteq S^{\prime}$ and $S_{0}$ is the unique maximal torus of $\overline{M_{v}\left(k_{s}\right)}$. Since $G_{v}\left(k_{s}\right)$ normalises $N$ and $K, G_{v}\left(k_{s}\right)$ normalises $N$ and $S_{0}$, so $G_{v}\left(k_{s}\right) \subseteq H$; it follows that $H_{v}\left(k_{s}\right)=G_{v}\left(k_{s}\right)$.

Let $H_{1}=H / N S_{0}$ and let $\pi: H \rightarrow H_{1}$ be the canonical projection. We wish to find $\lambda_{1} \in Y_{k_{s}}\left(H_{1}\right)$ such that $\lambda_{1}$ properly destabilizes $v$ over $k_{s}$ and $P_{\lambda_{1}}\left(H_{1}\right)$ is $k$-defined. Note that no nontrivial $k_{s}$-defined cocharacter of $H_{1}$ fixes $v$; for if $0 \neq \lambda_{1} \in Y_{k_{s}}\left(H_{1}\right)$ fixes $v$ then by Lemma 4.1, there exist $n \in \mathbb{N}$ and $\lambda \in Y_{k_{s}}(M)$ such that $\pi \circ \lambda=n \lambda_{1}$, and $\langle\operatorname{Im}(\lambda) \cup S\rangle$ is a $k_{s}$-defined torus of $\overline{G_{v}\left(k_{s}\right)}$ that properly contains $S$, contradicting the maximality of $S$. Clearly, $\left(H_{1}\right)_{v}^{0}$ is nilpotent with unique maximal torus $S_{1}^{\prime}:=\pi\left(S^{\prime}\right)$. Since $H^{0}=C_{G}\left(S_{0}\right)^{0}, H\left(k_{s}\right) \cdot v$ is not cocharacterclosed over $k_{s}$, by Theorem 2.7(ii) and [Bate et al. 2015, Corollary 5.3]. Hence $H_{1}\left(k_{s}\right) \cdot v$ is not cocharacter-closed over $k_{s}$ (Lemma 4.1). Let $\lambda_{1} \in Y_{k_{s}}\left(H_{1}\right)$ such that $\lambda_{1}$ destabilizes $v$. By Lemmas 2.8 and 4.1, we can assume $\lambda_{1}$ does not properly destabilize $v$ over $\bar{k}$. Therefore, there exists $u \in R_{u}\left(P_{\lambda_{1}}\left(H_{1}\right)\right)$ such that $u \cdot \lambda_{1}$ fixes $v$; then $u \cdot \lambda_{1}$ must be a cocharacter of $S_{1}^{\prime}$. It follows that $\Sigma_{v, k_{s}}\left(H_{1}\right) \subseteq \Delta_{k_{s}}\left(T_{1}, \mathbb{Q}\right)$, where $T_{1}$ is a fixed maximal torus of $H_{1}$ that contains $S_{1}^{\prime}$. Note that $T_{1}$ and $S_{1}^{\prime}$ need not be $k$-defined, or even $k_{s}$-defined. As $H_{1}\left(k_{s}\right) \cdot v$ is not cocharacter-closed over $k_{s}, \Sigma_{v, k_{s}}\left(H_{1}\right)$ is not completely reducible (Lemma 3.5(ii)). Now $\Sigma_{v, k_{s}}\left(H_{1}\right)$ is stabilized by $\Gamma$ and by $\left(H_{1}\right)_{v}\left(k_{s}\right)$, so it follows from Theorem 3.9 that $\Sigma_{v, k_{s}}\left(H_{1}\right)$ contains a $\Gamma$-fixed and $\left(H_{1}\right)_{v}\left(k_{s}\right)$-fixed point $x_{1}$. We can write $x_{1}=\zeta\left(\mu_{1}\right)$ for some $\mu_{1} \in Y_{k_{s}}\left(H_{1}\right)$. Then $\mu_{1}$ destabilizes $v$ but does not fix $v$; moreover, $P_{\mu_{1}}\left(H_{1}^{0}\right)$ is $\Gamma$-stable and is normalised by $\left(H_{1}\right)_{v}\left(k_{s}\right)$. In particular, $P_{\mu_{1}}\left(H_{1}^{0}\right)$ is $k$-defined.

By Lemma 4.1, there exist $n \in \mathbb{N}$ and $\mu \in Y_{k_{s}}(M)$ such that $\pi \circ \mu=n \mu_{1}$ and $\mu$ destabilizes $v$; note that $\mu$ does not fix $v$, because $\mu_{1}$ does not. The map $\pi$ gives a bijection between the parabolic subgroups of $M^{0}$ and the parabolic subgroups of $H_{1}^{0}$. So $P_{\mu}\left(M^{0}\right)$ is $\Gamma$-stable - because $P_{\mu_{1}}\left(H_{1}^{0}\right)$ is - and hence is defined over $k$. As $\pi\left(H_{v}\left(k_{s}\right)\right)$ is contained in $\left(H_{1}\right)_{v}\left(k_{s}\right)$ and $\left(H_{1}\right)_{v}\left(k_{s}\right)$ normalises $P_{\mu_{1}}\left(H_{1}^{0}\right), H_{v}\left(k_{s}\right)$ normalises $P_{\mu}\left(M^{0}\right)$.

After replacing $\mu$ if necessary with an $R_{u}\left(P_{\mu}\left(H^{0}\right)\right)\left(k_{s}\right)$-conjugate of $\mu$, we can assume that $\mu$ is a cocharacter of a $k$-defined maximal torus $T$ of $P_{\mu}\left(H^{0}\right)$. Let 
$\mu^{(1)}, \ldots, \mu^{(r)}$ be the $\Gamma$-conjugates of $\mu$. These are cocharacters of $T$, so they all commute with each other. Set $\sigma=\sum_{i=1}^{r} \mu^{(i)}$, a $k$-defined cocharacter of $T$. Note that $\sigma$ centralizes $S=S_{0} S_{2}$. Now $\pi \circ \sigma$ destabilizes $v$ but does not fix $v$ (since $\pi \circ \sigma \neq 0$ ), so $\sigma$ does not fix $v$. This implies by [Bate et al. 2015, Lemma 2.8] that $\sigma$ properly destabilizes $v$ over $k_{s}$ for $G$. Since $H_{v}\left(k_{s}\right)$ is $\Gamma$-stable and fixes $\zeta(\mu), H_{v}\left(k_{s}\right)$ fixes $\zeta\left(\mu^{(i)}\right)$ for all $1 \leq i \leq r$. It follows from Lemma 4.4 that $H_{v}\left(k_{s}\right)$ fixes $\zeta(\sigma)$ : that is, for all $h \in H_{v}\left(k_{s}\right)$, there exists $u \in R_{u}\left(P_{\sigma}\left(H^{0}\right)\right)\left(k_{s}\right)$ such that $h \cdot \sigma=u \cdot \sigma$. Hence $P_{\sigma}\left(G^{0}\right)$ is normalised by $H_{v}\left(k_{s}\right)=G_{v}\left(k_{s}\right)$. This completes the proof of (i).

Parts (ii) and (iii) now follow as in the proof of Theorem 1.1 (there is no need to reduce to the case when $v$ is a $k$-point in (iii) because we already assume this).

Remark 4.6. It can be shown that Theorem 1.3(iii) actually holds without the assumption that $v$ is a $k$-point. Here is a sketch of the proof. It is enough to prove that Levi ascent holds. Without loss, assume $S$ is a maximal $k$-defined torus of $G_{v}$. As in the proof of Theorem 1.1(iii), we replace $v$ with a $k$-point $w$ of a $k$-defined $G$-variety $W$, with the property that $\Sigma_{w, k_{s}} \subseteq \Sigma_{v, k_{s}}$ and $\Sigma_{w, k_{s}}=\Sigma_{v, k_{s}}$. By the arguments of [Bate et al. 2015, Section 6], we can assume that $S$ and $N$ fix $w$. Suppose $G(k) \cdot v$ is not cocharacter-closed over $k$. Then $G(k) \cdot w$ is not cocharacter-closed over $k$, so $L\left(k_{s}\right) \cdot w$ is not cocharacter-closed over $k_{s}$, by Galois descent and split Levi ascent. It follows that $H_{1}\left(k_{s}\right) \cdot w$ is not cocharacter-closed over $k_{s}$, where $H_{1}$ is defined as in the proof of Theorem 1.3. We do not know whether hypotheses (a) and (b) of Theorem 1.3 hold for $w$. The key point, however, that makes the proof of Theorem 1.3(i) work is that $\Sigma_{v, k_{s}}\left(H_{1}\right)$ is contained in a single apartment of $\Delta_{k_{s}}\left(H_{1}, \mathbb{Q}\right)$. The analogous property holds for $\Sigma_{w, k_{s}}\left(H_{1}\right)$ since $\Sigma_{w, k_{s}}\left(H_{1}\right) \subseteq \Sigma_{v, k_{s}}\left(H_{1}\right)$. Hence Galois ascent holds and $H_{1}(k) \cdot w$ is not cocharacter-closed over $k$. Then $H_{1}(k) \cdot v$ is not cocharacter-closed over $k$, and the result follows.

\section{Applications to $G$-complete reducibility}

Many of the constructions in this paper, and in the key references [Bate et al. 2013; 2012; 2015], were inspired originally by the study of Serre's notion of $G$-complete reducibility for subgroups of $G$. We refer the reader to [Serre 2005] and [Bate et al. 2005] for a thorough introduction to the theory. We simply record the basic definition here:

Definition 5.1. A subgroup $H$ of $G$ is said to be $G$-completely reducible over $k$ if whenever $H$ is contained in a $k$-defined R-parabolic subgroup $P$ of $G$, there exists a $k$-defined R-Levi subgroup $L$ of $P$ containing $H$. (We do not assume that $H$ is $k$-defined.) 
Theorem 5.2 ([Bate et al. 2015, Theorem 9.3]). Let $H$ be a subgroup of $G$ and let $\boldsymbol{h} \in H^{n}$ be a generic tuple of $H$ (see [Bate et al. 2013, Definition 5.4]). Then $H$ is $G$-completely reducible over $k$ if and only if $G(k) \cdot \boldsymbol{h}$ is cocharacter-closed over $k$, where $G$ acts on $G^{n}$ by simultaneous conjugation.

Theorem 5.2 allows us to prove results about $G$-complete reducibility over $k$ using our results on geometric invariant theory. If $G$ is connected, and $\boldsymbol{h} \in G^{n}$ is a generic tuple for a subgroup $H$ of $G$, then $\Sigma_{\boldsymbol{h}}$ is a subcomplex of $\Delta_{G}(\mathbb{Q})$, since for any $\lambda \in Y, \lambda$ destabilizes $\boldsymbol{h}$ if and only if $H \subseteq P_{\lambda}$; this means that we are in the territory of Theorem 1.1.

Proof of Theorem 1.4. Let $\boldsymbol{h}$ be a generic tuple of $H$. Then $\Sigma_{\boldsymbol{h}, k_{s}}$ is a subcomplex of $\Delta_{G, k_{s}}$, and $C_{G}(H)=G_{\boldsymbol{h}}$. The result now follows from Theorems 5.2 and 1.1(iii).

This theory has a counterpart for Lie subalgebras of $\mathfrak{g}:=\operatorname{Lie}(G)$. The basic definitions and results were covered for algebraically closed fields in [McNinch 2007] and [Bate et al. 2011, Section 3.3], but the extension to arbitrary fields is straightforward (cf. [Bate et al. 2011, Remark 4.16]).

Definition 5.3. A Lie subalgebra $\mathfrak{h}$ of $\mathfrak{g}$ is $G$-completely reducible over $k$ if whenever $P$ is a $k$-defined parabolic subgroup of $G$ such that $\mathfrak{h} \subseteq \operatorname{Lie}(P)$, there exists a $k$-defined Levi subgroup $L$ of $P$ such that $\mathfrak{h} \subseteq \operatorname{Lie}(L)$. (We do not assume that $\mathfrak{h}$ is $k$-defined.)

The group $G$ acts on $\mathfrak{g}^{n}$ via the simultaneous adjoint action for any $n \in \mathbb{N}$. The next result follows from [Bate et al. 2011, Lemma 3.8] and the arguments in the proofs of [Bate et al. 2011, Theorems 4.12(iii)] (cf. [Bate et al. 2011, Theorem 3.10(iii)]).

Theorem 5.4. Let $\mathfrak{h}$ be a Lie subalgebra of $\mathfrak{g}$ and let $\boldsymbol{h} \in \mathfrak{h}^{n}$ such that the components of $\boldsymbol{h}$ generate $\mathfrak{h}$ as a Lie algebra. Then $\mathfrak{h}$ is $G$-completely reducible over $k$ if and only if $G(k) \cdot \boldsymbol{h}$ is cocharacter-closed over $k$.

We now give the applications of our earlier results to $G$-complete reducibility over $k$ for Lie algebras.

Theorem 5.5. Let $\mathfrak{h}$ be a Lie subalgebra of $\mathfrak{g}$.

(i) Suppose $\mathfrak{h}$ is $k$-defined, and let $k^{\prime} / k$ be a separable algebraic extension. Then $\mathfrak{h}$ is $G$-completely reducible over $k^{\prime}$ if and only if $\mathfrak{h}$ is $G$-completely reducible over $k$.

(ii) Let $S$ be a $k$-defined torus of $C_{G}(\mathfrak{h})$ and set $L=C_{G}(S)$. Then $\mathfrak{h}$ is $G$-completely reducible over $k$ if and only if $\mathfrak{h}$ is $L$-completely reducible over $k$.

Proof. Pick $\boldsymbol{h} \in \mathfrak{h}^{n}$ for some $n \in \mathbb{N}$ such that the components of $\boldsymbol{h}$ generate $\mathfrak{h}$ as a Lie algebra. If $\mathfrak{h}$ is $k$-defined then we can assume that $\boldsymbol{h} \in \mathfrak{h}(k)^{n}$. Part (i) now follows from Theorems 5.4 and 1.1(ii), and part (ii) from Theorems 5.4 and 1.1(iii). 


\section{Acknowledgments}

The authors acknowledge the financial support of EPSRC Grant EP/L005328/1 and of Marsden Grants UOC1009 and UOA1021. Part of the research for this paper was carried out while the authors were staying at the Mathematical Research Institute Oberwolfach supported by the "Research in Pairs" programme. Also, parts of this paper were written during mutual visits to Auckland, Bochum and York. We thank the referee for a number of suggestions to improve the exposition.

\section{References}

[Bate et al. 2005] M. Bate, B. Martin, and G. Röhrle, "A geometric approach to complete reducibility", Invent. Math. 161:1 (2005), 177-218. MR 2007k:20101 Zbl 1092.20038

[Bate et al. 2009] M. Bate, B. Martin, and G. Röhrle, "On Tits' Centre Conjecture for fixed point subcomplexes”, C. R. Math. Acad. Sci. Paris 347:7-8 (2009), 353-356. MR 2010i:20049 Zbl 1223.20041

[Bate et al. 2011] M. Bate, B. Martin, G. Röhrle, and R. Tange, "Complete reducibility and conjugacy classes of tuples in algebraic groups and Lie algebras”, Math. Z. 269:3-4 (2011), 809-832. MR 2860266 Zbl 1242.20058

[Bate et al. 2012] M. Bate, B. Martin, and G. Röhrle, "The strong Centre Conjecture: An invariant theory approach”, J. Algebra 372 (2012), 505-530. MR 2990024 Zbl 1291.51007

[Bate et al. 2013] M. Bate, B. Martin, G. Röhrle, and R. Tange, "Closed orbits and uniform $S$-instability in geometric invariant theory”, Trans. Amer. Math. Soc. 365:7 (2013), 3643-3673. MR 3042598 Zbl 1284.20051

[Bate et al. 2015] M. Bate, S. Herpel, B. Martin, and G. Röhrle, "Cocharacter closure and the rational Hilbert-Mumford Theorem”, preprint, 2015. arXiv 1411.7849

[Borel 1991] A. Borel, Linear algebraic groups, 2nd ed., Graduate Texts in Mathematics 126, Springer, New York, 1991. MR 92d:20001 Zbl 0726.20030

[Conrad et al. 2010] B. Conrad, O. Gabber, and G. Prasad, Pseudo-reductive groups, New Mathematical Monographs 17, Cambridge Univ. Press, 2010. MR 2011k:20093 Zbl 1216.20038

[Hesselink 1978] W. H. Hesselink, "Uniform instability in reductive groups", J. Reine Angew. Math. 303/304 (1978), 74-96. MR 81a:14022 Zbl 0386.20020

[Humphreys 1975] J. E. Humphreys, Linear algebraic groups, Graduate Texts in Mathematics 21, Springer, New York, 1975. MR 53 \#633 Zbl 0325.20039

[Kempf 1978] G. R. Kempf, "Instability in invariant theory", Ann. of Math. (2) 108:2 (1978), 299-316. MR 80c:20057 Zbl 0406.14031

[Leeb and Ramos-Cuevas 2011] B. Leeb and C. Ramos-Cuevas, "The center conjecture for spherical buildings of types $F_{4}$ and $E_{6}$ ”, Geom. Funct. Anal. 21:3 (2011), 525-559. MR 2012i:51012 Zbl 1232.51008

[McNinch 2007] G. McNinch, "Completely reducible Lie subalgebras", Transform. Groups 12:1 (2007), 127-135. MR 2008a:17022 Zbl 1126.17007

[Mühlherr and Tits 2006] B. Mühlherr and J. Tits, "The center conjecture for non-exceptional buildings”, J. Algebra 300:2 (2006), 687-706. MR 2007e:51018 Zbl 1101.51004

[Mühlherr and Weiss 2013] B. Mühlherr and R. M. Weiss, "Receding polar regions of a spherical building and the center conjecture", Ann. Inst. Fourier (Grenoble) 63:2 (2013), 479-513. MR 3112519 Zbl 1296.20032 
[Ramos-Cuevas 2013] C. Ramos-Cuevas, "The center conjecture for thick spherical buildings", Geom. Dedicata 166 (2013), 349-407. MR 3101173 Zbl 1283.51006

[Rousseau 1978] G. Rousseau, "Immeubles sphériques et théorie des invariants", C. R. Acad. Sci. Paris Sér. A-B 286:5 (1978), A247-A250. MR 58 \#22063 Zbl 0375.14013

[Serre 1997] J.-P. Serre, "La notion de complète réductibilité dans les immeubles sphériques et les groupes réductifs", résumé, 1997. Séminaire au Collège de France.

[Serre 2005] J.-P. Serre, "Complète réductibilité", exposé no. 932, 195-217 in Séminaire Bourbaki, 2003/2004, Astérisque 299, Société Mathématique de France, Paris, 2005. MR 2006d:20084 Zbl 1156.20313

[Springer 1998] T. A. Springer, Linear algebraic groups, 2nd ed., Progress in Mathematics 9, Birkhäuser, Boston, 1998. MR 99h:20075 Zbl 0927.20024

[Tits 1974] J. Tits, Buildings of spherical type and finite BN-pairs, Lecture Notes in Mathematics 386, Springer, Berlin, 1974. MR 57 \#9866 Zbl 0295.20047

[Waterhouse 1979] W. C. Waterhouse, Introduction to affine group schemes, Graduate Texts in Mathematics 66, Springer, New York, 1979. MR 82e:14003 Zbl 0442.14017

Received June 1, 2015. Revised September 27, 2015.

Michael Bate

DEPARTMENT OF MATHEMATICS

UNIVERSITY OF YORK

YORK YO10 5DD

UNITED KINGDOM

michael.bate@york.ac.uk

Sebastian Herpel

FAKULTÄT FÜR MATHEMATIK

RUHR-UNIVERSITÄT BOCHUM

UNIVERSITÄTSSTRASSE 150

D-44780 BOCHUM

GERMANY

sebastian.herpel@rub.de

BENJAMIN MARTIN

DEPARTMENT OF MATHEMATICS

University of ABERdeEn, King's College

FRASER NOBLE BUILDING

ABERDEEN AB24 3UE

UNITED KINGDOM

b.martin@abdn.ac.uk

GERHARD RÖHRLE

FAKULTÄT FÜR MATHEMATIK

RUHR-UNIVERSITÄT BOCHUM

UNIVERSITÄTSSTRASSE 150

D-44780 BOCHUM

GERMANY

gerhard.roehrle@rub.de 


\title{
PACIFIC JOURNAL OF MATHEMATICS
}

\author{
msp.org/pjm
}

Founded in 1951 by E. F. Beckenbach (1906-1982) and F. Wolf (1904-1989)

\section{EDITORS}

Don Blasius (Managing Editor)

Department of Mathematics

University of California

Los Angeles, CA 90095-1555

blasius@math.ucla.edu

\author{
Paul Balmer \\ Department of Mathematics \\ University of California \\ Los Angeles, CA 90095-1555 \\ balmer@math.ucla.edu \\ Robert Finn \\ Department of Mathematics \\ Stanford University \\ Stanford, CA 94305-2125 \\ finn@math.stanford.edu \\ Sorin Popa \\ Department of Mathematics \\ University of California \\ Los Angeles, CA 90095-1555 \\ popa@math.ucla.edu
}

\author{
Vyjayanthi Chari \\ Department of Mathematics \\ University of California \\ Riverside, CA 92521-0135 \\ chari@math.ucr.edu \\ Kefeng Liu \\ Department of Mathematics \\ University of California \\ Los Angeles, CA 90095-1555 \\ liu@math.ucla.edu \\ Jie Qing \\ Department of Mathematics \\ University of California \\ Santa Cruz, CA 95064 \\ qing@ cats.ucsc.edu
}

\section{PRODUCTION}

Silvio Levy, Scientific Editor, production@msp.org

\section{SUPPORTING INSTITUTIONS}

ACADEMIA SINICA, TAIPEI

CALIFORNIA INST. OF TECHNOLOGY

INST. DE MATEMÁTICA PURA E APLICADA

KEIO UNIVERSITY

MATH. SCIENCES RESEARCH INSTITUTE

NEW MEXICO STATE UNIV.

OREGON STATE UNIV.

\author{
STANFORD UNIVERSITY \\ UNIV. OF BRITISH COLUMBIA \\ UNIV. OF CALIFORNIA, BERKELEY \\ UNIV. OF CALIFORNIA, DAVIS \\ UNIV. OF CALIFORNIA, LOS ANGELES \\ UNIV. OF CALIFORNIA, RIVERSIDE \\ UNIV. OF CALIFORNIA, SAN DIEGO \\ UNIV. OF CALIF., SANTA BARBARA
}

\author{
Daryl Cooper \\ Department of Mathematics \\ University of California \\ Santa Barbara, CA 93106-3080 \\ cooper@math.ucsb.edu \\ Jiang-Hua Lu \\ Department of Mathematics \\ The University of Hong Kong \\ Pokfulam Rd., Hong Kong \\ jhlu@maths.hku.hk \\ Paul Yang \\ Department of Mathematics \\ Princeton University \\ Princeton NJ 08544-1000 \\ yang@math.princeton.edu
}

These supporting institutions contribute to the cost of publication of this Journal, but they are not owners or publishers and have no responsibility for its contents or policies.

See inside back cover or msp.org/pjm for submission instructions.

The subscription price for 2015 is US \$420/year for the electronic version, and \$570/year for print and electronic.

Subscriptions, requests for back issues and changes of subscribers address should be sent to Pacific Journal of Mathematics, P.O. Box 4163, Berkeley, CA 94704-0163, U.S.A. The Pacific Journal of Mathematics is indexed by Mathematical Reviews, Zentralblatt MATH, PASCAL CNRS Index, Referativnyi Zhurnal, Current Mathematical Publications and Web of Knowledge (Science Citation Index).

The Pacific Journal of Mathematics (ISSN 0030-8730) at the University of California, c/o Department of Mathematics, 798 Evans Hall \#3840, Berkeley, CA 94720-3840, is published twelve times a year. Periodical rate postage paid at Berkeley, CA 94704, and additional mailing offices. POSTMASTER: send address changes to Pacific Journal of Mathematics, P.O. Box 4163, Berkeley, CA 94704-0163.

PJM peer review and production are managed by EditFLOW ${ }^{\circledR}$ from Mathematical Sciences Publishers.

\section{PUBLISHED BY}

\section{mathematical sciences publishers \\ nonprofit scientific publishing}

http://msp.org/

(C) 2015 Mathematical Sciences Publishers 


\title{
PACIFIC JOURNAL OF MATHEMATICS
}

\author{
Volume 279 No. 1-2 December 2015
}

In memoriam: Robert Steinberg

Robert Steinberg (1922-2014): In memoriam V. S. VARADARAJAN

Cellularity of certain quantum endomorphism algebras

HENNING H. ANDERSEN, GUSTAV I. LEHRER and RUIBIN ZHANG

Lower bounds for essential dimensions in characteristic 2 via orthogonal representations ANTONIO BABIC and VLADIMIR CHERNOUSOV

Cocharacter-closure and spherical buildings

Michael Bate, Sebastian Herpel, Benjamin Martin and Gerhard RöHrLe

Embedding functor for classical groups and Brauer-Manin obstruction

Eva Bayer-Fluckiger, Ting-Yu LeE and Raman Parimala

On maximal tori of algebraic groups of type $G_{2}$

Constantin Beli, PhilipPe Gille and Ting-Yu LeE

On extensions of algebraic groups with finite quotient

MICHEL BRION

Essential dimension and error-correcting codes

SHANE CERNELE and ZiNOVy REICHSTEIN

Notes on the structure constants of Hecke algebras of induced representations of finite Chevalley groups

Charles W. CuRTis

Complements on disconnected reductive groups

FRANÇOIS DIGNE and JEAN MICHEL

Extending Hecke endomorphism algebras

Jie Du, Brian J. Parshall and LeOnard L. SCOTT

Products of partial normal subgroups

ELLEN HENKE

Lusztig induction and $\ell$-blocks of finite reductive groups

RADHA KESSAR and GUNTER MALLE

Free resolutions of some Schubert singularities

Manoj Kummini, Venkatramani Lakshmibai, Pramathanath Sastry and C. S. Seshadri

Free resolutions of some Schubert singularities in the Lagrangian Grassmannian

VenKatramani LAKSHMibai and ReUVEN HODGES

Distinguished unipotent elements and multiplicity-free subgroups of simple algebraic groups

Martin W. Liebeck, Gary M. Seitz and Donna M. Testerman

Action of longest element on a Hecke algebra cell module

GEORGE LUSZTIG

Generic stabilisers for actions of reductive groups

BENJAMIN MARTIN

On the equations defining affine algebraic groups

VLADIMIR L. POPOV

Smooth representations and Hecke modules in characteristic $p$

PETER SCHNEIDER

On CRDAHA and finite general linear and unitary groups

BHAMA SRINIVASAN

Weil representations of finite general linear groups and finite special linear groups PHAM HUU TIEP

The pro- $p$ Iwahori Hecke algebra of a reductive $p$-adic group, $\mathrm{V}$ (parabolic induction) MARIE-FRANCE VIGNÉRAS

Acknowledgement 